with a wave theory. He constructed such a theory, and it was correct, but rather than being an anticipation of later work, it was a rediscovery of a result published by Airy in 1838.

Raman's collaborator on the theory of the mirage was his talented nephew Pancharatnam, fated soon to die at a tragically early age. Venkataraman gives a detailed account of Pancharatnam's ideas on the interference of polarized light. Only now are we beginning to appreciate their originality and depth - for example his anticipation of the geometric phases now in fashion.

These and many other areas of physics are explained with elegance and clarity. The author is a theoretical physicist with a flair for simple and direct exposition. Sometimes more sophisticated mathematical treatments are merited, and these are given in separate sections so as not to interrupt the main narrative.

Raman seems to have been a difficult and obstinate man. His dealings with administrators and bureaucrats, men of narrow vision compared to him, were stormy, and he made enemies among his colleagues. He became embroiled in controversy and resigned in rage, first from his chair in Calcutta and then from the directorship of the Indian Institute of Science in Bangalore. Finally he founded his own Raman Research Institute in Bangalore. That Institute continues today as a jewel in the crown of Indian science, a tribute to Raman and his successor Radhakrishnan. Venkataraman discusses these matters in great detail and with sensitivity. His book makes us appreciate how unusual Raman must have been to produce such an abundance of creative science, first in the stifling colonial atmosphere of British India, and then in the turbulent struggle for independence.

Michael Berry is a Professor in the H.H. Wills Physics Laboratory, University of Bristol, Tyndall Avenue, Bristol BS8 1TL, UK.

- Oxford University Press will also be the distributor of Raman's collected works, in six volumes and under the title Scientific Papers of C.V. Raman. The books will become available in July-August, price $£ 25, \$ 49.95$ per volume.

\section{Well does He?}

\section{Robert M. May}

Does God Play Dice? The Mathematics of Chaos. By Ian Stewart. Basil Blackwell: 1989. Pp.317. £15. To be published in the United States in May, c. $\$ 19.95$.

READERS OF Nature will recognize Ian Stewart as the person whose News and Views articles continually demonstrate that it is indeed possible to convey the excitement - and the substance - of advances on the frontiers of pure mathematics to a wide audience. Does God Play Dice? is aimed even more widely, to the reading public at large.

The book opens with a far-reaching proposition (with which I agree). In earlier times, the world was seen as unpredictable, governed at best by capricious deities. Over the past few centuries, the newtonian revolution has given us the opposite view, with most things seeming to be predictable provided one has the right rules or equations and enough computing power. But we may be at the start of another swing of the pendulum, with the recognition that many simple and fully deterministic rules give essentially unpredictable or chaotic dynamical behaviour.

In his second and third chapters, Stewart sets the stage with succinct yet lucid accounts of the newtonian paradigm ("Equations for Everything") and of the view that chance or random behaviour derives from complexity ("The Laws of Error"). Poincaré ("The Last Universalist") is seen as bestriding a watershed. Refining the newtonian tradition, he sought qualitative, geometrical ways of understanding the behaviour of systems of differential equations. In the process, he gave birth to the discipline of topology, and initiated the long transition from the classical mechanics of the nineteenth century (with its increasingly elegant solutions of those subsets of equations that could be solved elegantly) to the dynamical systems theory of today. As translated by Stewart, Poincaré recognized what are now called strange attractors in a simplified version of the three-body problem:

When one tries to depict the figure formed by these two curves and their infinity of intersections [one gets] a kind of net, web, or infinitely tight mesh; neither of the two curves can ever cross itself, but must fold back on itself in a very complex way in order to cross the links of the web infinitely many times. One is struck with the complexity of this figure that I am not even attempting to draw.

Stewart launches from this springboard into a lively account of contemporary work on chaos: Smale's formal analysis of strange attractors; the chaotic behaviour (and beautiful regularities) of the quadratic map and other first-order difference equations with 'one hump'; Lorenz's already-classic set of three differential equations as a metaphor for unpredictability in weather systems; the Voyager data and Wisdom's computations on the chaotic tumbling of Hyperion, a moon of Saturn; and much else.

Fractals, the Mandelbrot set and other fashionable topics are also covered, but are clearly tied to the central theme enunciated in the opening chapter. In particular, Stewart gives an excellent, intuitive account of the relation between fractals and chaos (it is not widely appreciated that the geometrical distinction between smooth forms, such as circles and spheres, and rough forms, such as fractal objects, is formally akin to the distinction between the familiar attractors of classical dynamics and the strange attractors of chaos; a fractal dimension can be associated with any given strange attractor, characterizing qualitative features of the dynamics).

Stewart's book will inevitably be compared with James Gleick's deservedly successful Chaos: Making a New Science (Viking, 1987). Both books do a good job of presenting complicated material in a way that is engaging, accurate and accessible to the uninitiated. Both also convey the fascination of the subject. Gleick spends more time on personalities, and on the excitement of the chase. This, I think, may make his account a better read for the layperson, and means that it captures the existential, higgledy-piggledy way science really advances. Gleick's understandable affection for colourful personalities sometimes leads to discrepancies between his 'programme credits' and the perceptions of those in the discipline about who did what first, which has led to some rough reviews by professionals (for example that in Nature 330, 293; 1987).

Although racily written, with lots of staccato paragraphs such as "Simple. Elegant. Elusive." or just "No.", Stewart's book concentrates rather less on people and more on the science itself. Even so, its programme credits are not impeccable. The basic catalogue of the various periodic orbits for generically quadratic maps is attributed to Sharkovskii, whereas among the many independent discoverers I believe the neglected Myrberg comes first; the bifurcation diagram for this map was first published, and its properties of selfsimilarity on smaller and smaller scales noted, by Oster and myself in American Naturalist (110, 573-599; 1976), but here the diagram is as usual called the Feigenbaum tree, permitting a multilingual 'figtree' pun. Such attributions of priority matter only to the players. The play itself is what really counts and here Stewart is, not surprisingly, stronger than Gleick on the mathematics, particularly on how the various aspects of the subject fit together.

In a thought-provoking final chapter Stewart speculates upon the implications of our still-emerging understanding of chaos. Observing that "order can breed its own kind of chaos", he returns to the celebrated exchange between Einstein and Born, and suggests "The question is not so much whether God plays dice, but how God plays dice".

Robert M. May is a Royal Society Research Professor in the Department of Zoology, University of Oxford, South Parks Road, Oxford OX1 3PU, UK, and at Imperial College, London. 